\title{
Immunodeficiency and Cancer
}

National Cancer Institute

\section{Source}

National Cancer Institute. Immunodeficiency and Cancer. NCI Thesaurus. Code C19610.

Investigations into the relationship between immune function and cancer.

Immunodeficient individuals are less able to mount an attack on cancerous cells. 